\title{
Corrugated horn design for HFI on PLANCK
}

Cite as: AIP Conference Proceedings 616, 282 (2002); https://doi.org/10.1063/1.1475645

Published Online: 06 May 2002

J. A. Murphy, R. Colgan, E. Gleeson, B. Maffei, C. O'Sullivan, and P. A. R. Ade

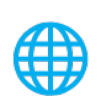

View Online

\section{ARTICLES YOU MAY BE INTERESTED IN}

Use of high sensitivity bolometers for astronomy: Planck high frequency instrument AIP Conference Proceedings 605, 571 (2002); https://doi.org/10.1063/1.1457711

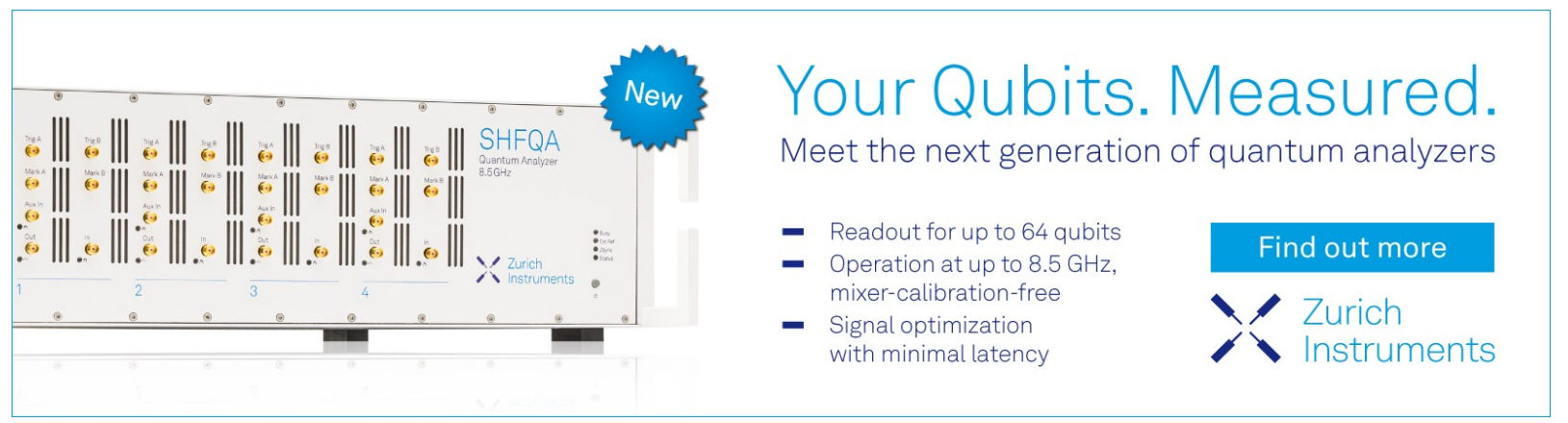




\title{
Corrugated Horn Design for HFI on PLANCK
}

 \\ $\mathrm{Ade}^{2}$ \\ ${ }^{I}$ National University of Ireland, Maynooth, Co. Kildare, Ireland \\ ${ }^{2}$ University of Wales, Cardiff, CF24 3YB, UK.
}

\begin{abstract}
In this paper we report on the back-to-back corrugated feed horn design for the High Frequency Instrument on the PLANCK Surveyor. Special single moded Gaussian profiled horns have been developed for the 4 lowest frequency channels $(100 \mathrm{GHz}, 150 \mathrm{GHz}, 217 \mathrm{GHz} \& 353 \mathrm{GHz})$. These feed structures produce very pure Gaussian radiation patterns with sidelobe levels reduced well below $-30 \mathrm{~dB}$. Similar few moded horn antennas are being proposed for the highest frequency channels $(545 \mathrm{GHz}$ and $850 \mathrm{GHz})$ to give non-diffraction limited performance with high throughput. The modelling of the horns uses a rigorous electromagentic mode matching technique that is found to give good agreement with measurement.
\end{abstract}

\section{INTRODUCTION}

On the PLANCK Surveyor the telescope is directly fed by two arrays of horn antenna feeds with no front-end optics to condition the radiation patterns of the individual horns. The sidelobe levels of the illuminating horn feeds on the telescope must therefore be sufficiently low so that strong background sources do not cause contamination of the faint signal. Simple single moded and multi-moded horn structures such as conical horns and Winston cones are inadequate in this application to reach the level of rejection required without compromising the resolution. Therefore, more complex corrugated horn designs that produce much lower level sidelobes have to be considered $[1,2,3]$. It is important to have reliable modelling tools for predicting with a high degree of confidence the radiation patterns of such horns antennas, especially since we wish to profile the horn shape in order to optimize performance. We consider two alternate electromagnetic models as will be discussed in the next section of this paper.

Primarily we are concerned with the feed horn design of the HFI (High Frequency Instrument) on PLANCK [4]. Shown is Figure 1 is the basic quasi-optical concept for a single detector channel [5]. The corrugated front horn controls the beam pattern on the telescope, while the waveguide section controls the modes that can propagate. The back horn feeds the filter stack, which defines the frequency band and rejects any stray IR radiation. The $100 \mathrm{mK}$ horn is over-moded and so can accept all incident radiation. From a modelling point of view this arrangement can be idealized as a black body cavity illuminating the back horn (see Figure 2(a)) [6]. The high frequency channels on the HFI employ multi-moded horns with the number of modes being determined by the wave-guide filter properties (guide radius and corrugation depths). We can apply the same electromagnetic models both to the lower frequency single mode and the higher frequency multi-mode horns [7]. This procedure and related issues such as bandwidth and radiation characteristics are considered in detail in later sections of the paper.

\section{MODELLING OF HORN ANTENNAS}

Two electromagnetic methods are available for modelling horn antennas. The first based on based on modematching, and involving complex scattering matrix calculations, is accurate with exact horn profile definition needed for the analysis. The second approach based on hybrid modes is more approximate in that the corrugated waveguide wall is regarded as a non-uniform impedance surface. 


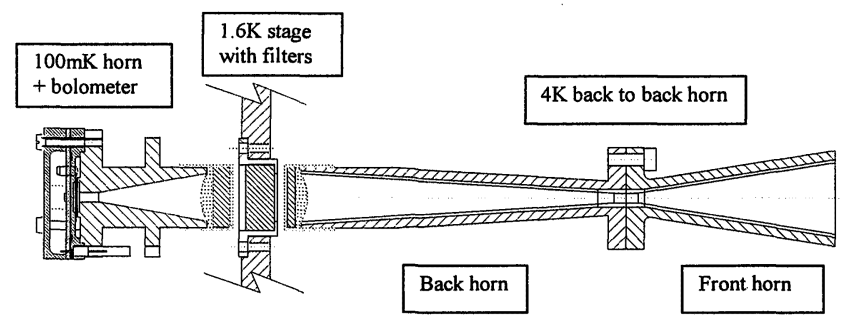

Figure 1: Back to back horn design

The mode matching approach assumes that the waveguide can be regarded as a sequence of very short smooth walled segments making up the slots and ridges of the corrugations [8]. Since the structures are cylindrically symmetric pure cylindrical waveguide modes are used for describing the propagation of the electromagnetic radiation. At each corrugation step account is taken of all of the scattering that occurs at the sharp transition in the guide width (see Figure 2(b)). One of the main advantages of the approach is that horns with an arbitrary shaped profile can be handled readily and the fields in the actual horn flare can be modelled right up to the horn aperture. The radiation pattern of the horn can then be written in terms of either a fully coherent or a partially coherent sum of the far field patterns of the modes in the horn aperture (depending on whether the horn is single moded or multimoded). These features have been incorporated into a software program called SCATTER.

In the case of the surface impedance model (appropriate when there are many corrugations per wavelength) an average surface impedance can be assumed [1]. The true of modes of propagation in that case turn out to be hybrid mode combinations of TE and TM modes (true TE or TM modes do not satisfy the boundary conditions). Solving the waveguide equation for these hybrid modes is rather complex compared to smooth walled guide (see Figure $3, \&$ [1]). As the modes approach the cut-off condition that the waveguide number $\beta$ approaches zero, the so called HE modes become pure TM, while the corresponding EH modes become TE-like. The modes also have a high frequency cut-off which is approached as the corrugation slots get deeper for a given guide radius, as can be seen in Figure 3. Being modes of propagation these hybrid modes are not scattered in a corrugated waveguide. Furthermore, for a simple conical corrugated horn we can make the approximation that essentially the mode propagates into the horn without scattering and attains a spherical phase error with radius of curvature equal to the horn axial length.

In corrugated conical horns with resonant grooves $(\lambda / 4$ deep) balanced hybrid modes are formed with tapered fields at the aperture, as illustrated in Figure 4. Thus, in a perfect non scattering horn the modal radiation patterns are not coherently related to each other, and so to obtain the overall beam pattern for a multi-moded horn the individual modal patterns must be added in quadrature. However, if scattering is expected to occur (as in a shaped overmoded corrugated horn) we have to apply the mode matching software. The application of hybrid modes is thus limited to conical horns and the simple analysis of waveguide filters.



(a)

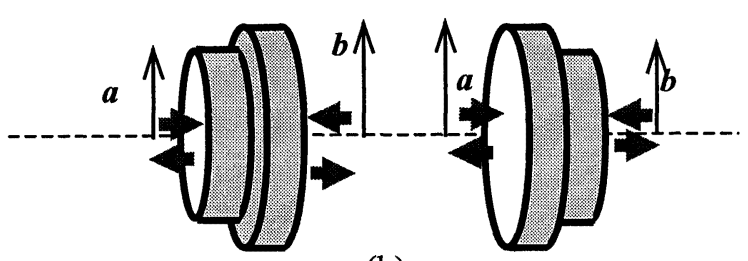

(b)

FIGURE 2. (a). Idealisation of Back-to-back Feedhorn Design \& (b) waveguide step details 




$r_{i} / r_{0}=0.7$

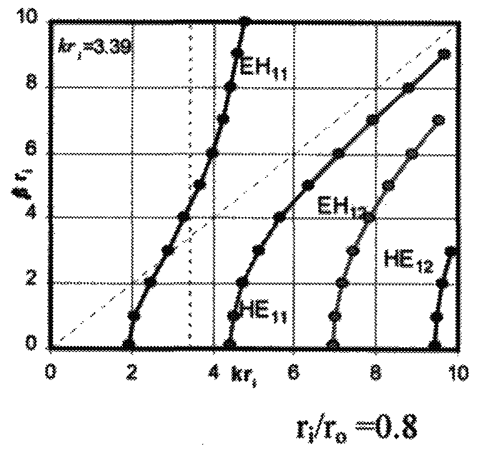

Figure 3: Dispersion curves for modes of azimuthal order 1 in a waveguide filter showing $\mathrm{kr}_{\mathrm{i}}=3.39$.

(a)

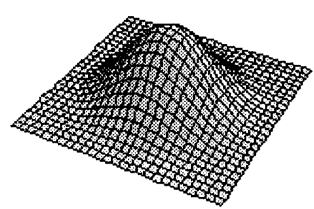

(c)



(b)

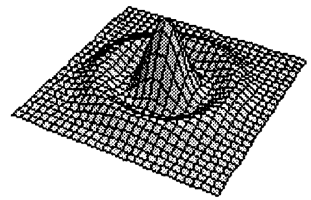

$(d)$

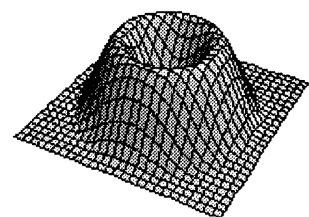

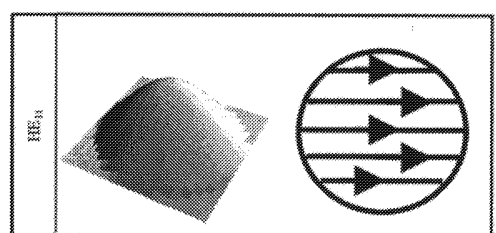

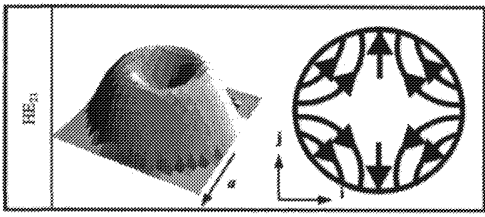

Figure 4: Aperture field intensities for (a) $\mathrm{HE}_{11}$, (b) $\mathrm{HE}_{12}$, (c) $\mathrm{EH}_{12}$ and (d) $\mathrm{HE}_{21}$ balanced hybrid modes.

\section{SINGLE MODE CORRUGATED HORN DESIGN}

Corrugated feed horns have several advantages for application to microwave background experiments. Such horns produce linearly polarised symmetric beam patterns with low sidelobe levels. However, the PLANCK demands on edge taper make it hard to achieve acceptable resolution with conical corrugated horns as the sidelobe levels are higher than the required $-30 \mathrm{~dB}$ at the primary reflector. This has led to the development of so called profiled Gaussian horns which produce a beam pattern with an extremely high purity Gaussian beam (see Figure $5 \&$ $[9,2])$. Sidelobe levels well below $-30 \mathrm{~dB}$ can be achieved, and there is a high level of agreement between the mode matching model predictions and the measurements as is clear in Figure 6. However, the horns tend to have a narrower bandwidth (in terms of beam pattern quality) than linearly tapered conical corrugated horns.

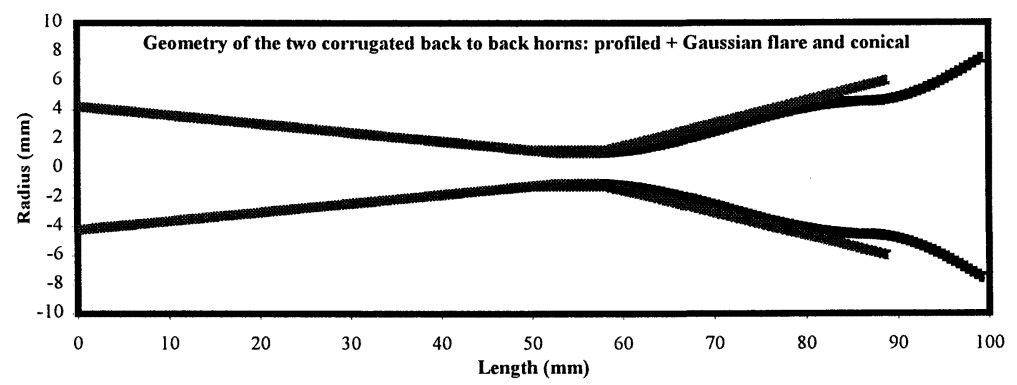

Figure 5: Superposition of the geometry of the two corrugated prototype horns. 


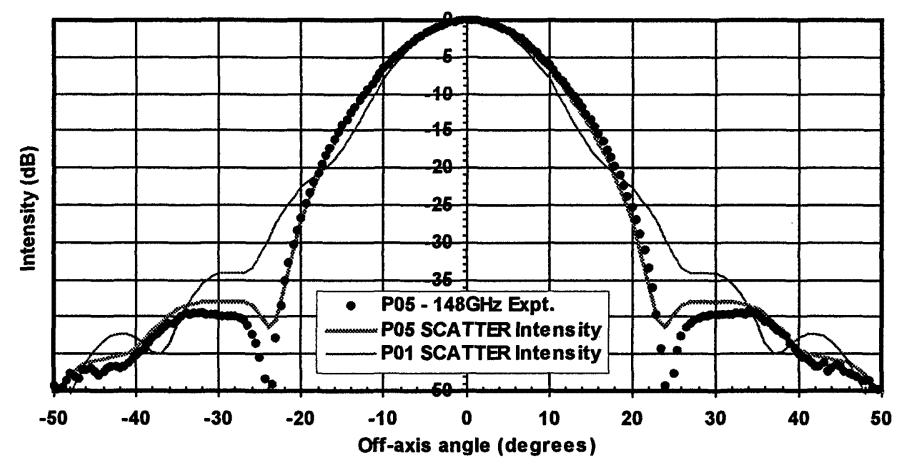

Figure 6: Far-field beam pattern (experimental and modal matching).

The phase centre is estimated by fitting a spherical phase correction to the far-field phase error, which arises because the far field calculations are referenced to the horn aperture. Empirically, by running several examples, it was determined that the horn shape influences how far down the horn the phase centre is located, with this position being adjustable to some degree by varying the opening angle of the Gaussian flare on the horn (see Table 1 and Figure 9, which summarises the horn design achieved by B. Maffei).

Experimental measurement of the transmission profile over a wide frequency range using an FTS arrangement at QMW shows that the transmission pass bands are limited for both conical and flared corrugated horns (see Figure 7). This is not experienced with smooth walled horns, and implies in fact, for multi-mode corrugated horn operation, one needs wave-guide filter slots that are not too deep otherwise the fundamental $\mathrm{HE}_{11}$ is cut-off. However, if the slots are too shallow the $\mathrm{EH}_{1}$ mode can propagate (there is not a sharp cut-off for the band). Evidence for this is illustrated in Figure 9 in which the edge of the pass-band for the $143 \mathrm{GHz}$ horn was examined as a function of slot depth. Careful modelling using the mode matching approach was therefore required to optimize the wave-guide filter operation for either single mode operation (where the wave-guide acts as the high pass filter for the system) or multi-mode operation (in which the pass-band is defined by a quasi-optical filter behind the back to back horn, as shown in Figure 1).

TABLE 1. List of Single Mode Horn Parameters

\begin{tabular}{cccc}
\hline Frequency & Aperture Radius & Horn Length & Phase Centre Position \\
\hline $100 \mathrm{GHz}$ & $7.81 \mathrm{~mm}$ & $88.9 \mathrm{~mm}$ & $4.4 \mathrm{~mm}$ \\
$143 \mathrm{GHz}$ & $5.49 \mathrm{~mm}$ & $68.4 \mathrm{~mm}$ & $3.6 \mathrm{~mm}$ \\
$217 \mathrm{GHz}$ & $4.16 \mathrm{~mm}$ & $82.5 \mathrm{~mm}$ & $3.2 \mathrm{~mm}$ \\
$353 \mathrm{GHz}$ & $3.69 \mathrm{~mm}$ & $75.2 \mathrm{~mm}$ & $1.8 \mathrm{~mm}$
\end{tabular}

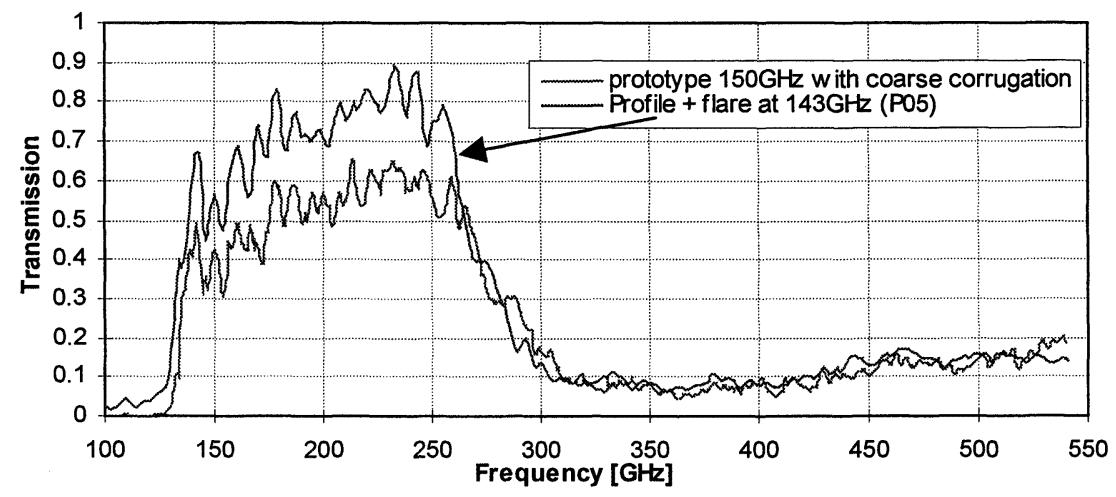

Figure 8: Transmission of corrugated waveguide filter shows band for fundamental mode is limited. 



$100 \mathrm{GHz}$
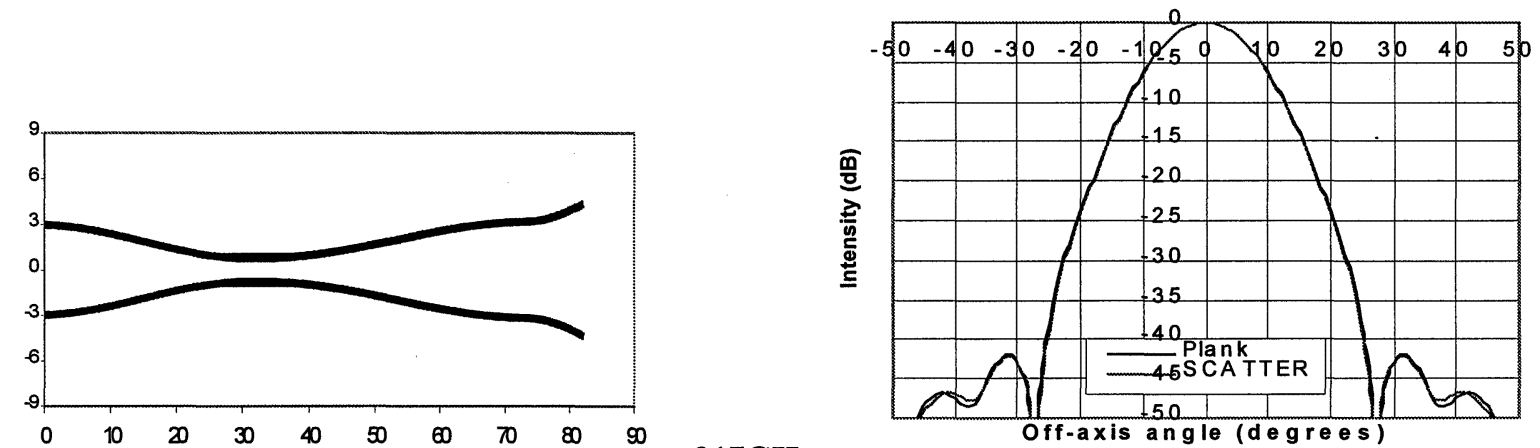

$217 \mathrm{GHz}$
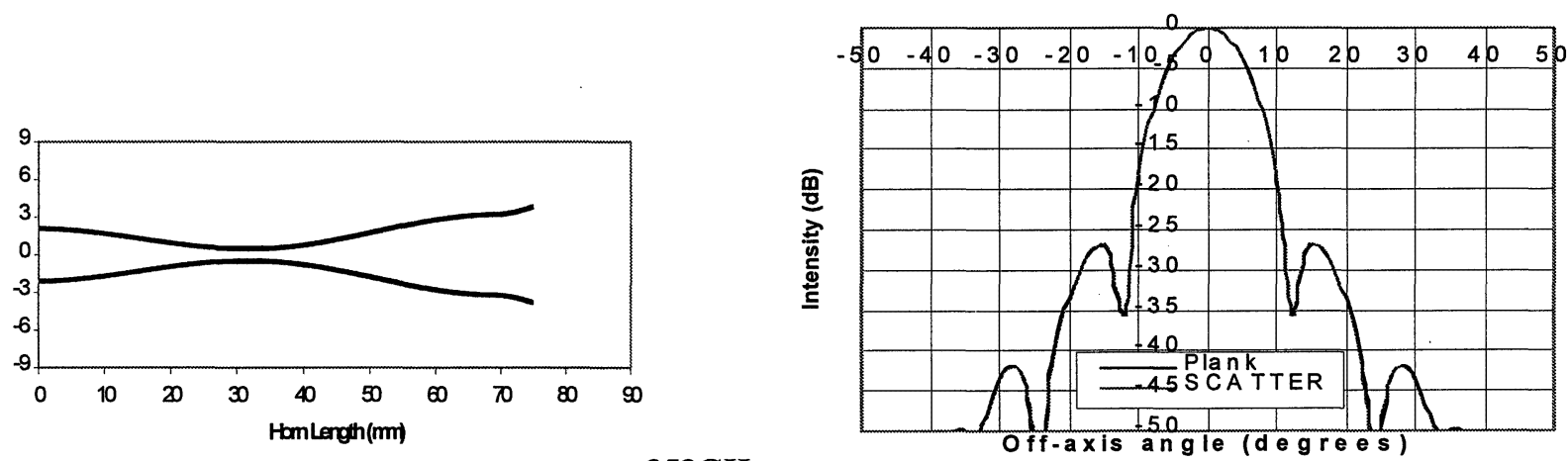

$353 \mathrm{GHz}$

Figure 7: Horn Profiles (exaggerated, distances in $\mathrm{mm}$ ) \& Beam Patterns with two model predictions

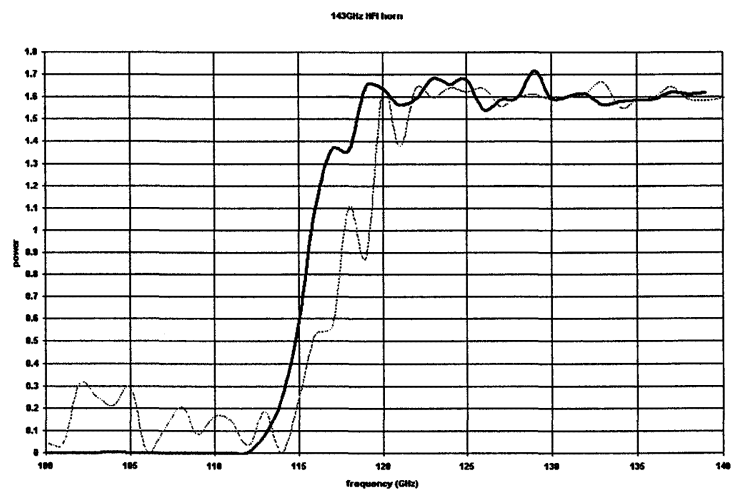

Figure 9. Model predictions for transmission at band edge (deep slots - thick line, shallow slots - thin line) 


\section{MULTI-MODE HORN DESIGN}

Multi-moded horns provide high throughput when diffraction limited resolution is not required on a telescope. This is the case for the two high frequency channels on PLANCK (at $545 \mathrm{GHz}$ and $857 \mathrm{GHz}$ ). Such overmoded horns produce somewhat broader beams on the sky than would be the case if the system fed by the same horns were single moded. Because of extreme sensitivity to background contamination we still of course require very low edge taper and spill over levels here, as was the case for the single mode channels. In the case of the multi-moded horns it is effectively the waveguide filter that determines which modes can propagate over a certain frequency band. Figure 10 shows the low frequency and high frequency cut-offs for the hybrid modes that propagate in corrugated waveguides.

In order to experimentally verify the proposed multi-mode horn designs for PLANCK a $150 \mathrm{GHz}$ scale mode of the proposed $545 \mathrm{GHz}$ design was manufactured and tested at QMW. A conical taper was chosen and by performing measurements at $150 \mathrm{GHz}$ higher accuracy could be achieved, which is important to test the predictive reliability of the modelling software and the assumptions made about the number of modes that can propagate (see Table $2 \&$ Fig. 11). By also testing the same horn at $200 \mathrm{GHz}$ we mimic the operation of the PLANCK $850 \mathrm{GHz}$ channel, in which a relatively large number of modes should be allowed to propagate (see Table $2 \&$ Fig. 12).

In general, as illustrated by Figs. $11 \& 12$, for a given horn shape and aperture radius the measured far field radiation pattern of the horn (which illuminates the telescope) becomes broader also and tends to become top-hat like for long horns and many modes. Also shown on the plot are the model predictions for the horn. By comparng Figs. $11 \& 12$ one notes that as more modes are added for a given horn geometry, by simply operating such a horn at progressively higher frequencies, the beam pattern tends to have about the same width (this is, of course, expected from consideration of conservation of throughput for such a system). There is relatively good agreement between model and measurement, but with some indication of transmission loss for the highest order modes that can propagate.

TABLE 2. List of propagating pure TE/TM modes (for the SCATTER software) for prototype muilti-moded horn at $150 \mathrm{GHz}$, cut-off wavenumbers for $r_{i}=1.62 \mathrm{~mm}$.

\begin{tabular}{lcc}
\hline Frequency/GHz & $\boldsymbol{K} \boldsymbol{r}_{\boldsymbol{I}}$ & Propagating Modes \\
\hline 100 & 3.39 & TE11, TM01, TE21 \\
150 & 5.09 & TE11, TM01, TE21, TM11, TE01, TE31 \\
200 & 6.78 & TE1 1,TM01,TE21,TM11,TE01,TE31,TM02, TM21,TE12,TM31, TE51, TE22
\end{tabular}


Figure 10: Low frequency \& High frequency cut-off charts for lower order hybrid modes.

High frequency cut-off chart. For $\mathrm{r}_{\mathrm{i}} / \mathrm{r}_{\mathrm{o}}=0.7, \mathrm{HE}_{11}$ cutoff at $\mathrm{kr} \approx 10$ 


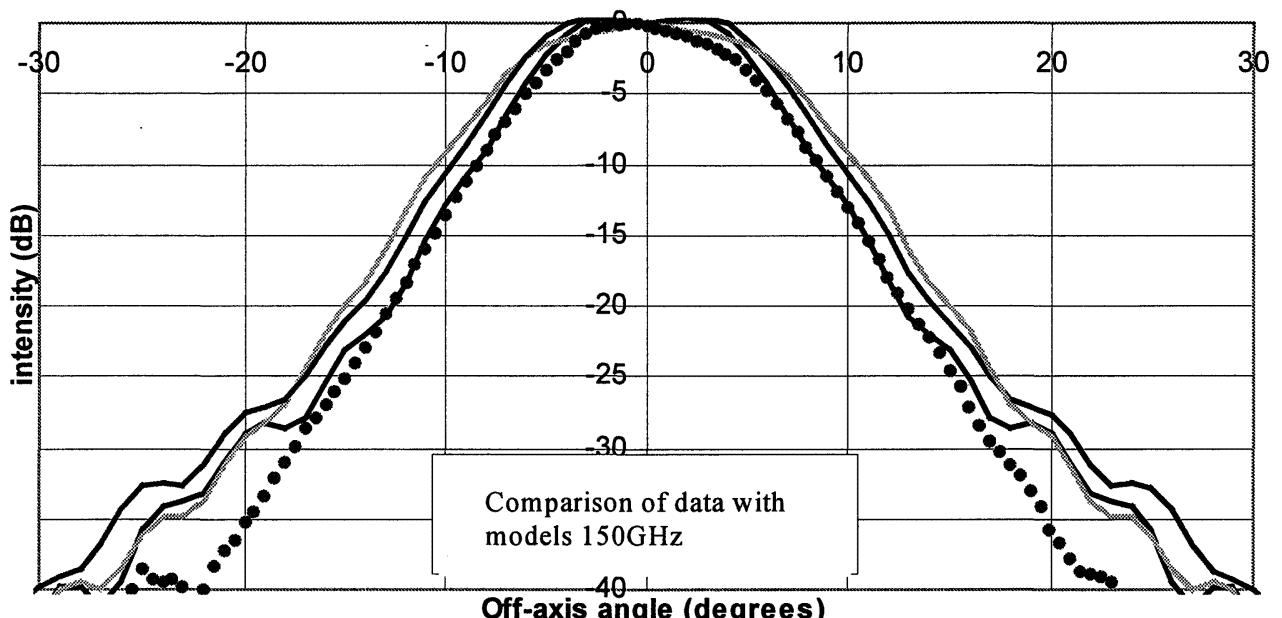

Figure 11. Beam pattern of prototype scale mode horn for $150 \mathrm{GHz}$ (test data - dots, hybrid model - light grey, mode matching - black $(n=2 \& n=3))$.

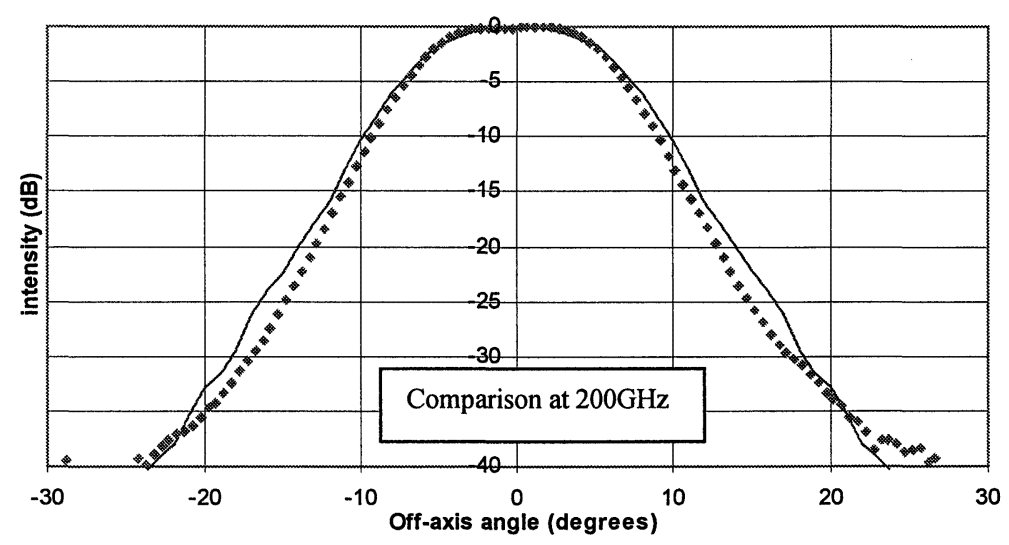

Figure 12: Beam pattern at $200 \mathrm{GHz}$ for multi-moded scale horn (test data - dots, model - line)

The phase centre of a multimoded horn is an ill-defined concept since the fields are only partially coherent and each spatial mode has a separate centre of phase curvature. In terms of operation on the telescope what is most important is either maximising the gain of the telescope or minimising the FWHM ( $3 \mathrm{~dB}$ resolution). The quality of the radiation pattern may also be important since a multi-moded horn beam results from summing several modal fields and can lead to the main telescope beam having significant structure. Thus, we can calculate for a perfect telescope (oversized with negligible edge taper and aberrational effects) how the beam shape or the on-axis gain on the sky varies as the horn aperture is moved relative to the true focal plane of the telescope (see Figure 13). In this way the "phase-centre" of a multi-moded horn can be calculated.

\section{CONCLUSIONS}

We summarize the design strategy for the HFI horn array for PLANCK as follows. Profiled corrugated horns are to be used for single moded horn design. Such horns offer very low edge taper levels compared with conical corrugated horns leading to optmised resolution. Futhermore, the position of phase centre can be adjusted by varying opening angle of the flare.

The definition of the multi-moded horns is now being finalized. Here again profiled horns are to be used to control position of the "phase centre" although the effect on the beam pattern edge taper is less pronounced. Aberrations appear to lead so some distortion and have to be included in the determination of resolution [10]. 


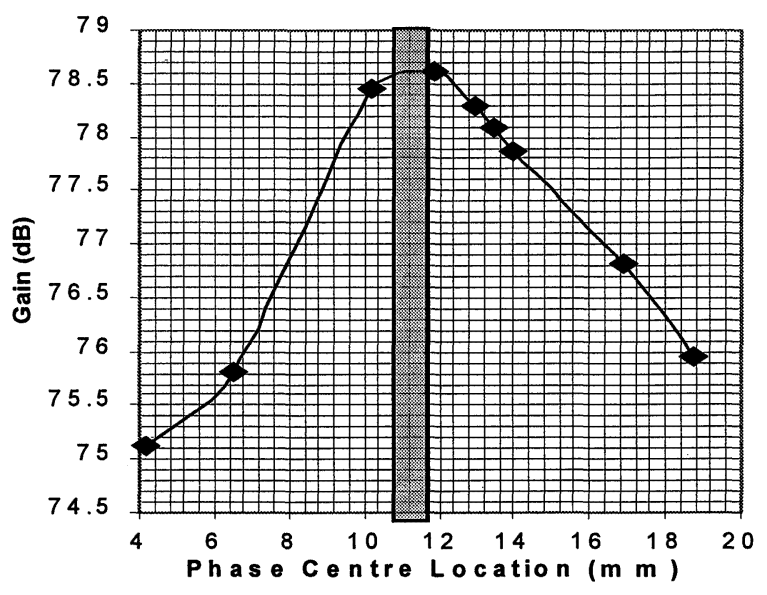

Figure 13. Gain of $545 \mathrm{GHz}$ multi-mode horn as function of aperture position with respect to telescope focal plane.

\section{ACKNOWLEDGMENTS}

The authors wish to acknowledge the financial assistance of Enterprise Ireland.

\section{REFERENCES}

1. Clarricoats P. J. B. and Olver A. D., Corrugated Horns for Microwave Antennas, IEE Electromagnetic Waves Series 18, Peter Perigrinus, London,1984, pp 20-57.

2. Maffei B. et al, International Journal of Infrared and Millimeter Waves 21, 2023-2034 (2000).

3. Padman R. and Murphy J.A., Infrared Physics 31, 441-446 (1991).

4. Lamarre J-M. et al, Astro. Letters and Communications, 37, 161-170 (2000).

5. Church S. E. et al, "A compact high-efficiency feed structure for Cosmic Microwave Background Astronomy at Millimetre Wavelengths", Proceedings $30^{\text {th }}$ ESLAB Symposium Submillimetre and Far-Infrared Space Instrumentation, ESA SP-388, edited by E.J. Rolfe. 1996, pp 77-80.

6. Murphy J. A. and Padman R., Infrared Physics 31, 291-299 (1991).

7. Murphy J. A. et al, Infrared Physics and Technology, 41, 515-528 (2001) .

8. Olver A. D. et al, Microwave Horns and Feeds, IEE Electromagnetic Waves Series 39, IEEE Press, New York, 1994, pp 100-122.

9. del Rio C. et al, IEEE Transactions Antennas \& Propagation AP-47, 1440-1448 (1999).

10. Yurchenko V. B., Murphy J.A. and Lamarre J-M, International Journal of Infrared and Millimeter Waves 22, 173-184 (2001). 